\title{
Long-Term Oncologic Outcomes of Minimally Invasive Partial Nephrectomy for Renal-Cell Carcinoma
}

\author{
Anudeep Mukkamala, BA, Chang He, MS, Alon Z. Weizer, MD, MS, Khaled S. Hafez, MD, \\ David C. Miller, MD, MPH, Jeffrey S. Montgomery, MD, MHSA, and J. Stuart Wolf, Jr., MD, FACS
}

\begin{abstract}
Purpose: To report the long-term oncologic outcomes and survival estimates associated with minimally invasive partial nephrectomy (MIPN) and to determine factors associated with those outcomes and survival estimates.

Patients and Methods: A single-institution, retrospective review was performed on all patients undergoing MIPN for renal-cell carcinoma between 1998 and 2011 with minimum 1-year follow-up. Bivariate and multivariate analyses were performed to assess associations between demographic, perioperative, and tumor factors with recurrence and survival. Survival was estimated using the Kaplan-Meier method.

Results: Of 417 patients undergoing MIPN, median overall and oncologic follow-up were 3.3 and 2.9 years, respectively. The mean patient age was 63 years (standard deviation [SD] \pm 13.4 ). The mean tumor size was $2.9 \mathrm{~cm}(\mathrm{SD} \pm 1.48)$. Only $6.7 \%$ of patients had a pathologic stage $\mathrm{T}_{2}$ or greater. There was only one cancerrelated death. Estimates for overall survival at 2, 5, and 10 years were 95.6\%, 89.1\%, and 70.7\%, respectively. Estimates for recurrence-free survival (any recurrence) at 2, 5, and 10 years were $98.2 \%, 93.5 \%$, and 88.3\%, respectively. On multivariate analysis, only tumor stage was associated with recurrence, and only patient age and American Society of Anesthesiologists score were associated with overall survival. Technical aspects of the procedure, such as positive margins or use of enucleation, did not influence recurrence or survival.

Conclusions: Cancer recurrence after MIPN, in a cohort of patients with mostly $\mathrm{pT}_{1}$ tumors, is rare. Recurrence and overall survival are associated with nonmodifiable factors rather than technical ones.
\end{abstract}

\section{Introduction}

$\mathbf{F}$ OR SMALL RENAL PARENCHYMAL TUMORS, partial nephrectomy $(\mathrm{PN})$ has become a standard of care even in the presence of a normal contralateral kidney. Underused a decade ago, ${ }^{1}$ more recent data suggest increasing application of partial as opposed to radical nephrectomy. ${ }^{2,3}$ Although open surgical PN is the gold standard approach to PN, particularly for complex tumors, ${ }^{4,5}$ minimally invasive PN (MIPN) appears to have comparable short-term functional and oncologic outcomes. ${ }^{6,7}$ As such, MIPN is also increasing in utilization. ${ }^{8}$ This has occurred without widespread confirmation of long-term oncologic efficacy, with only Lane and associates $^{9-11}$ reporting oncologic outcomes of MIPN after 5,7 , and 10 years.

Consequently, the aim of this study is to identify factors associated with long-term overall and cancer-related survival after MIPN, with the hope of informing preoperative patient selection and intraoperative decision making, and to potentially direct future research on the oncologic efficacy of MIPN.

\section{Patients and Methods}

All patients undergoing MIPN (includes standard, handassisted, and robotic-assisted laparoscopy) at our institution between November 1998 and September 2011 were included in our Institutional Review Board approved and prospectively maintained database. A total of 480 patients underwent MIPN for pathologically confirmed renal-cell carcinoma; those with benign pathology were excluded from this analysis. Of these patients, a minimum 1-year follow-up was available in 417 patients. Long-term oncologic data were obtained and analyzed retrospectively from medical records, radiographic reports, tumor and death registries, and contact with patients, their families, and referring physicians' offices. In patients with bilateral disease managed with staged procedures $(n=3)$, only the first operation was included. Patients with preexisting metastatic disease were excluded from the study.

Our follow-up surveillance protocol comprised regular history and physical examinations together with laboratory testing, chest radiography, and computed tomography (CT)

Department of Urology, University of Michigan Hospitals and Health Centers, Ann Arbor, Michigan. 
or magnetic resonance imaging (MRI) of the abdomen, generally following the recommendations of Lam and colleagues. ${ }^{12}$ For patients who underwent follow-up at another institution, reports from the referring physician were obtained and entered into our computerized database. All patients were accounted for in calculating overall survival $(n=417)$. In determining oncologic follow-up, chest radiography or crosssectional imaging was required. Only four patients lacked documentation of the imaging necessary for oncologic followup to estimate recurrence-free survival $(n=413)$, which included recurrence locally (anywhere in the ipsilateral kidney), regionally, or systemically. Cause of death was ascertained using a combination of sources: Physician note, hospital records, and the Social Security Death Index.

The surgical approach (i.e., standard laparoscopic partial nephrectomy [SLPN], hand-assisted laparoscopic partial nephrectomy [HALPN] and robot-assisted laparoscopic partial nephrectomy [RALPN] ), use of hilar clamping, and the performance of enucleation $v s$ sharp excision of tumor were determined based un tumor characteristics and surgeon preference.

The dependent variables for statistical analysis were overall survival, cancer-specific survival, and recurrence-free survival. Bivariate assessments were made with the log rank test, using the following independent variables: Patient age, American Society of Anesthesiologists (ASA) score, and preoperative estimated glomerular filtration rate (eGFR), (calculated using the Modification of Diet in Renal Disease equation ${ }^{13}$ ); tumor stage, grade, and size; surgical approach; enucleation vs sharp excision; ischemia time; and positive margin. We did not assess tumor cell type because of the small number and heterogeneity of nonclear cell types. We then fit Cox proportional hazards models after backward selection for the most parsimonious model to evaluate for association using those independent variables significant on bivariate analysis. Associations are shown as hazard ratios with $95 \%$ confidence intervals (CIs). Statistical analyses were performed using SAS 9.2 software (SAS Institute, Cary, NC).

\section{Results}

Among the 417 patients, the mean patient age was 63 years (standard deviation $[\mathrm{SD}] \pm 13.4), 62 \%$ were ASA 1 or 2 , and the mean pre-operative eGFR was 79 (SD \pm 22.4 ). Among the MIPN, there were 212 SLPN, 91 HALPN, and 114 RALPN. The mean tumor size was $2.9 \mathrm{~cm}$ (SD \pm 1.48$)$. Cell-types included clear-cell in $284(68 \%)$, papillary type 1 in $83(20 \%)$, chromophobe in $28(6.7 \%)$, papillary type 2 in $4(1.0 \%)$, and other or mixed in $18(4.3 \%)$. The tumor grade was 1 or 2 in $271(65 \%)$ patients, and 3 or 4 in the remainder. Most tumors were stage $\mathrm{pT}_{1 \mathrm{a}}(329,79 \%)$ or $\mathrm{pT}_{1 \mathrm{~b}}(59,14 \%)$, with only 28 $(6.7 \%)$ being tumor stage $\mathrm{pT}_{2}$ or greater (data missing in one patient). Of the procedures, $15 \%$ involved tumor enucleation and $85 \%$ were performed with sharp excision. The mean ischemia time was 21 minutes ( $\mathrm{SD} \pm 14.2)$. The final surgical margin was positive for tumor in $23(5.5 \%)$ patients.

The median overall follow-up was 3.3 years (interquartile range $[\mathrm{IQR}] 2.0-6.0$ years), including 120,70 , and 15 patients with follow-up more than 5, 7, and 10 years, respectively. There were 44 deaths overall. Only one patient died of renal cancer. Median oncologic follow-up was 2.9 years (IQR $1.5-4.8$ years). There were 17 recurrences $(4.1 \%)$, including $7(1.7 \%)$ in the ipsilateral kidney, 2 in the contralateral kidney, 3 in the lung, and 5 at other sites. Estimates for overall survival at 2, 5, and 10 years $(95 \% \mathrm{CI})$ were $95.6 \%(93.0$, 97.2), 89.1\% (84.5, 92.4), and 70.7\% (56.7, 80.9), respectively. Because there was only one death from renal cancer, we did not calculate cancer-specific survival. Estimates for recurrence-free survival (any recurrence) at 2, 5, and 10 years (95\% CI) were 98.2\% (96.1, 99.2), 93.5\% (88.8, 96.3), and $88.3 \%(79.6,93.5)$, respectively.

Bivariate analyses are summarized in Tables $1 \mathrm{~A}$ and $1 \mathrm{~B}$. Older patient age was associated with tumor recurrence, as were greater tumor stage, grade, and size, as well as the occurrence of a positive surgical margin. Notably, of the 23 patients with positive margin of resection, only 3 had any form of recurrence with 2 recurrences $(8.7 \%)$ specifically

Table 1A. Bivariate Analysis of Overall and Recurrence Free Survival

\begin{tabular}{|c|c|c|c|c|c|}
\hline Variable & Strata & $\begin{array}{c}\text { No. with } \\
\text { overall death } \\
\mathrm{n}=44\end{array}$ & P-value & $\begin{array}{c}\text { No. with } \\
\text { recurrence } \\
\mathrm{n}=17\end{array}$ & $\mathrm{P}$-value \\
\hline Tumor stage* & $\begin{array}{c}\mathrm{T}_{1 \mathrm{a}} \\
\mathrm{T}_{1 \mathrm{~b}} \\
\mathrm{~T}_{2} \text { or } \mathrm{T}_{3 \mathrm{a}}\end{array}$ & $\begin{array}{r}34 \\
5 \\
5\end{array}$ & 0.12 & $\begin{array}{l}7 \\
4 \\
6\end{array}$ & $<0.0001$ \\
\hline Tumor grade & $\begin{array}{l}1 \text { or } 2 \\
3 \text { or } 4\end{array}$ & $\begin{array}{l}28 \\
16\end{array}$ & 0.42 & $\begin{array}{r}7 \\
10\end{array}$ & 0.03 \\
\hline ASA & $\begin{array}{l}1 \text { or } 2 \\
3 \text { or } 4\end{array}$ & $\begin{array}{l}15 \\
29\end{array}$ & $<0.0001$ & $\begin{array}{l}9 \\
8\end{array}$ & 0.21 \\
\hline Approach & $\begin{array}{c}\text { RALPN } \\
\text { HALPN } \\
\text { SLPN }\end{array}$ & $\begin{array}{r}3 \\
19 \\
22\end{array}$ & 0.34 & $\begin{array}{r}2 \\
4 \\
11\end{array}$ & 0.81 \\
\hline Enucleation & $\begin{array}{l}\text { No } \\
\text { Yes }\end{array}$ & $\begin{array}{r}38 \\
6\end{array}$ & 0.55 & $\begin{array}{r}14 \\
3\end{array}$ & 0.21 \\
\hline Positive margin & $\begin{array}{l}\text { No } \\
\text { Yes }\end{array}$ & $\begin{array}{r}42 \\
2\end{array}$ & 0.97 & $\begin{array}{r}14 \\
3\end{array}$ & 0.047 \\
\hline
\end{tabular}

*Missing 1 patient.

ASA = American Society of Anesthesiologists; RALPN= robot-assisted laparoscopic partial nephrectomy; HALPN=hand-assisted laparoscopic partial nephrectomy; SLPN=standard laparoscopic partial nephrectomy. 
Table 1B. Bivariate Analysis of Overall and Recurrence-Free Survival

\begin{tabular}{|c|c|c|c|c|c|c|}
\hline \multirow[b]{2}{*}{ Variable (mean $\pm S D$ ) } & \multicolumn{2}{|c|}{ Overall survival } & \multirow[b]{2}{*}{$\mathrm{P}$-value } & \multicolumn{2}{|c|}{ Recurrence-free survival } & \multirow[b]{2}{*}{$\mathrm{P}$-value } \\
\hline & Death & No death & & Recurrence & No recurrence & \\
\hline $\begin{array}{l}\text { Preoperative eGFR } \\
\left(\mathrm{mL} / \mathrm{min} \text { per } 1.73 \mathrm{~m}^{2}\right)\end{array}$ & $71 \pm 4.0$ & $81 \pm 1.1$ & 0.04 & $73 \pm 6.5$ & $80 \pm 1.1$ & 0.41 \\
\hline Age (years) & $75 \pm 2.0$ & $62 \pm 0.7$ & $<0.0001$ & $72 \pm 2.3$ & $63 \pm 0.7$ & 0.048 \\
\hline Tumor size $(\mathrm{cm})$ & $2.9 \pm 0.2$ & $2.9 \pm 0.09$ & 0.16 & $3.9 \pm 0.5$ & $2.9 \pm 0.07$ & 0.001 \\
\hline Ischemia time (min) & $18 \pm 2.1$ & $21 \pm 0.7$ & 0.50 & $22 \pm 4.4$ & $21 \pm 0.7$ & 0.40 \\
\hline
\end{tabular}

$\mathrm{SD}=$ standard deviation; eGFR = estimated glomerular filtration rate.

located in the ipsilateral kidney. From a different perspective, of the seven patients with recurrence in the ipsilateral kidney, two $(29 \%)$ were associated with positive margins. In the three patients with positive surgical margins and recurrence, the median time to presentation of recurrence was 30 months. In the 20 patients with positive surgical margins but no recurrence, the median oncologic follow-up was 31 months. Overall survival was reduced in older patients and those with greater ASA score and lower preoperative eGFR. We also tested the association of tumor recurrence with overall survival, and there was none ( $P=0.92$, data not shown).

Figure 1 depicts the Kaplan-Meier survival curves for recurrence-free survival, stratified by tumor stage. Figure 2 depicts the Kaplan-Meier survival curves for overall survival, with Figure 2A stratified by age and Figure 2B stratified by ASA score. Of note, the survival estimate for overall survival reached $0 \%$ in the subcohort with ASA score 3 or 4 just beyond 12 years.

Results from the multivariate analyses are presented in Table 2. For recurrence, only tumor stage remained associated $(P<0.0001)$. The hazard ratios (relative to stage $\mathrm{pT}_{1 \mathrm{a}}$ ) were 4.81 (95\% CI, 1.37-16.97) for stage $\mathrm{pT}_{1 \mathrm{~b}}$ and 19.46 (95\% CI, 5.87-64.59) for stage $\mathrm{pT}_{2}$ or greater. Of the three factors associated with overall survival on bivariate analysis (patient age, ASA score, and preoperative eGFR), the last factor lost significance on multivariate analysis. The hazard ratio for age $(P<0.0002)$ was 1.05 (95\% CI, 1.02-1.08), with each additional year being associated with a $5 \%$ increase in the hazard of death. ASA score 3 or $4(P<0.0002)$ was associated with a hazard ratio of 3.53 (95\% CI, 1.81-6.85), relative to ASA score 1 or 2 .

\section{Discussion}

The management of small renal masses has evolved over the last decade from open surgical PN designed for maximum cancer control to include MIPN focused on the combined goals of oncologic efficacy, reduction of the duration and intensity of convalescence, and preservation of renal function. ${ }^{14} \mathrm{~A}$ number of groups have reported their perioperative and short-term experiences with MIPN, suggesting excellent oncologic efficacy, as well as low recurrence

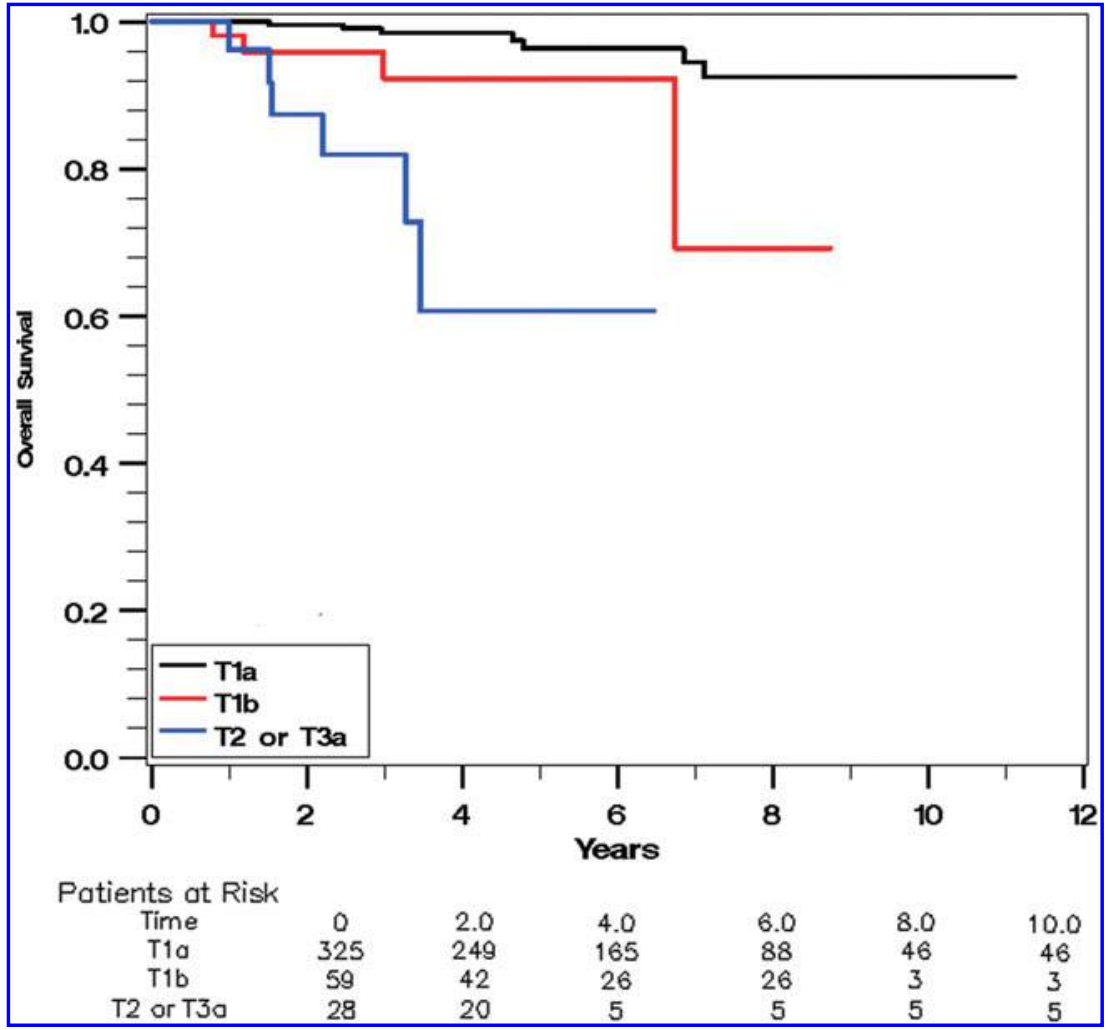

FIG. 1. Kaplan-Meier survival curve for recurrence-free survival, stratified by tumor stage. 
FIG. 2A. Kaplan-Meier survival curve for overall survival, stratified by age.

FIG. 2B. Kaplan-Meier survival curve for overall survival, stratified by American Society of Anesthesiologists score.
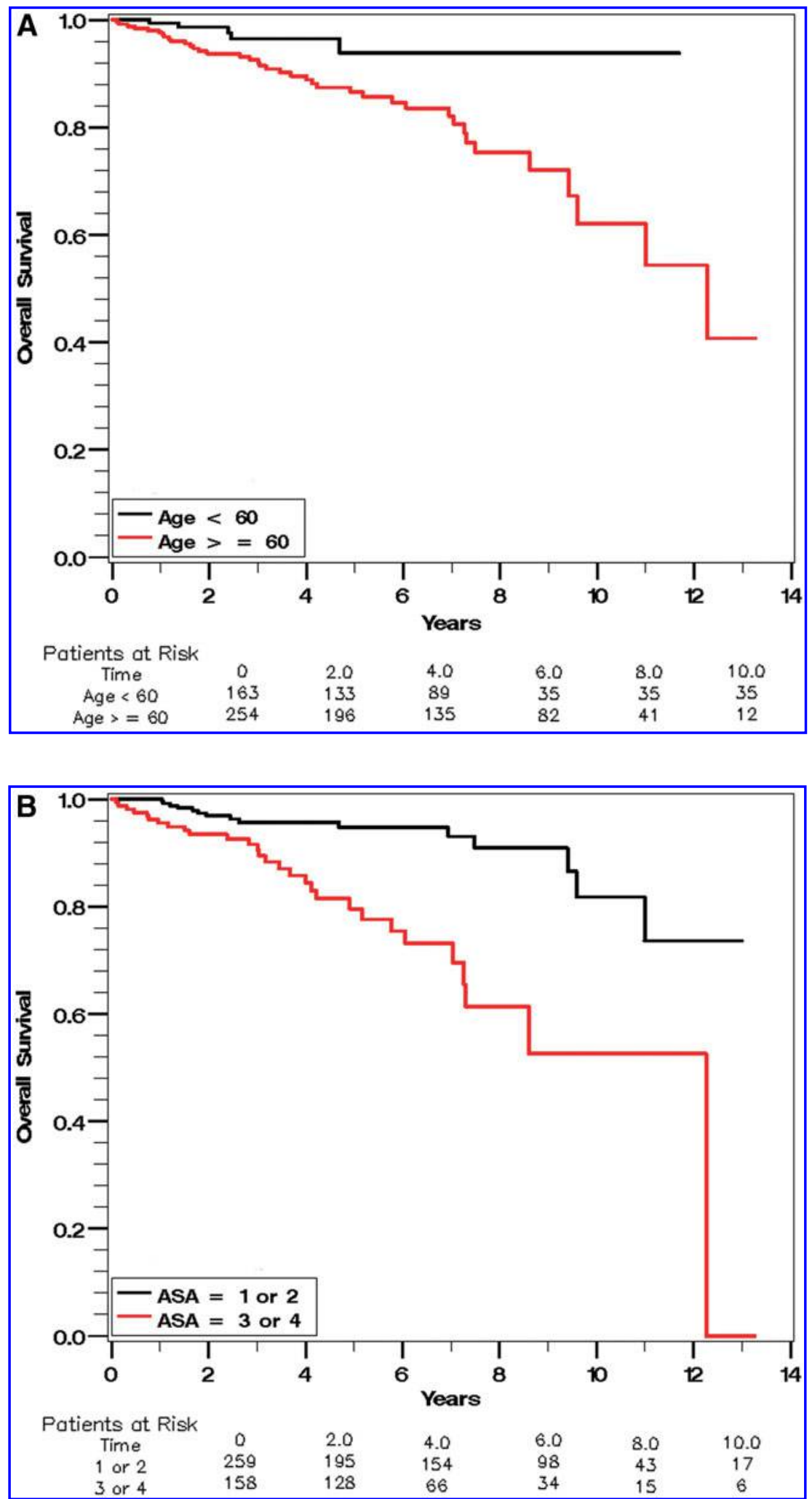

rates. ${ }^{15,16}$ Longer follow-up, however, is needed to confirm the oncologic efficacy of MIPN.

Some series that report oncologic follow-up in excess of 3 years are limited by the size of their cohorts. ${ }^{17,18}$ Lane and associates $^{11}$ recently reported a series that included 45 patients with more than 10 years follow-up after MIPN; overall survival was $78 \%$ at 10 years, and metastasis-free survival was $97 \%$. Using both laparoscopic and open surgical cohorts, these authors identified several independent predictors of overall survival and recurrence in the long term: Older age, larger tumor size, absolute indication, and Charlson comorbidity index. Because ASA score and Charlson 
Table 2. Multivariate Analysis of Overall and Recurrence Free Survival

\begin{tabular}{llcccc}
\hline Variable & Strata & $\begin{array}{c}\text { Overall death }(\mathrm{n}=44) \\
\text { Hazard ratio }(95 \% \text { CI })\end{array}$ & $\begin{array}{c}\text { P-value } \\
\text { Hazard ratio }(95 \% \text { CI })\end{array}$ & P-value \\
\hline Tumor Stage & $\mathrm{T}_{1 \mathrm{a}}{ }^{*}$ & $0.63(0.24,1.67)$ & 0.09 & $4.81(1.37,16.97)$ & $<0.0001$ \\
& $\mathrm{~T}_{1 \mathrm{~b}}$ & $2.43(0.93,6.37)$ & & $19.46(5.87,64.59)$ & \\
ASA & $\begin{array}{l}\mathrm{T}_{2} \text { or } \mathrm{T}_{3 \mathrm{a}} \\
1 \text { or } 2^{*}\end{array}$ & $3.53(1.81,6.85)$ & 0.0002 & & \\
Age & 3 or 4 & $1.05(1.02,1.08)$ & 0.0002 & & \\
\hline
\end{tabular}

*Reference group.

$\mathrm{CI}=$ confidence interval; ASA $=$ American Society of Anesthesiologists.

comorbidity index appear to predict mortality equally well, ${ }^{19,20}$ these findings are similar to ours with regard to overall survival. With regard to tumor recurrence, Lane and associates $^{11}$ found that surgery type was not associated with oncologic outcome, again similar to our finding that no technical aspects influenced recurrence-free survival.

Finally, our finding on multivariate analysis that positive surgical margins were not associated with tumor recurrence is consistent with a growing body of evidence. ${ }^{21-23}$ There are several possible explanations for the observation that positive surgical margins on pathologic examination of the resected tumor do not result in recurrence in many cases, including: Intraoperative fulguration of the tumor bed, ischemic damage to residual tumor from hemostatic sutures, retraction of normal parenchyma away from the tumor during tissue processing, and/or the appearance of a positive margin when the resection was adjacent to the tumor's pseudocapsule and in fact there is no tumor left in the patient. Alternatively, it may be that the duration needed for positive margins to produce a clinically noticeable tumor has not yet been achieved in most series.

In our cohort, characterized by relatively young and healthy patients with good renal function and small, low-risk tumors, the risks of recurrence and death were low, with estimated $88.3 \%$ recurrence-free survival and $70.7 \%$ overall survival at 10 years. There was only one cancer-related death. Of the 17 patients with recurrence, only 4 (3 with pulmonary metastases and 1 with abdominal lymph node metastases) had recurrence beyond the ipsilateral or contralateral kidney, adrenal gland, or a resectable solitary metastasis. As such, oncologic risk factors impacted only recurrence, and not survival. The excellent oncologic outcomes in our cohort do suggest great efficacy of MIPN, but they also suggest the possibility that at least some of these small renal cancers are being overtreated. In particular, competing causes of mortality are especially important, as illustrated by the finding of no survival beyond 12 years in patients with ASA score 3 or 4. We, like many other groups, are increasingly using active surveillance in patients with small renal masses and high competing risks of mortality. ${ }^{24}$ The excellent results of MIPN provide the context in which we should assess active surveillance strategies going forward.

The present study has several limitations including the selection bias for patients with unilateral, low-volume disease, the changes in surgery and practice patterns over the last decade, the varied learning curves of the different surgeons, errors in retrospective data extraction, and variability of patient follow-up. Despite these limitations, we think that this evalu- ation of the long-term outcomes of a large cohort of MIPN patients is crucial to better understanding this procedure.

\section{Conclusion}

To our knowledge, this is the first study in the literature to examine long-term ( $\geq 3$ years) oncologic outcomes after three different types of MIPN in a large population with each patient having at least 1 year of follow-up. Our data confirm that MIPN, in a cohort of mostly $\mathrm{pT}_{1}$ tumors, is associated with infrequent tumor recurrence even on long-term followup. Only tumor stage influences recurrence; the technical aspects of MIPN such as the surgical approach (SLPN, HALPN, or RALPN) or the method of resecting the tumor (enucleation or sharp excision) were not associated with recurrence. With limited impact of these small renal cancers on mortality, the clinician's operative decisions should be guided by the understanding that recurrence-free survival is influenced by tumor stage and that overall survival is influenced by patients' age and medical condition.

\section{Disclosure Statement}

Dr. Wolf is American Urological Association Chair, Practice Guidelines Committee, and an editorial consultant for Urology Times. For the remaining authors, no competing financial interests exist.

\section{References}

1. Miller DC, Hollingsworth JM, Hafez KS, et al. Partial nephrectomy for small renal masses: An emerging quality of care concern? J Urol 2006;175:853-858.

2. Ljungberg B. Contemporary status of open nephron-sparing surgery in renal cell carcinoma. Arch Ital Urol Androl 2009; 81:61-64.

3. Kheterpal E, Taneja SS. Partial nephrectomy: Contemporary outcomes, candidate selection, and surgical approach. Urol Clin North Am 2012;39:199-210.

4. Fergany AF, Saad IR, Woo L, Novick AC. Open partial nephrectomy for tumor in a solitary kidney: Experience with 400 cases. J Urol 2006;175:1630-1633.

5. Campbell SC, Novick AC, Belldegrun A, et al. Guideline for management of the clinical T1 renal mass. J Urol 2009; 182:1271-1279.

6. Gong EM, Orvieto MA, Zorn KC, et al. Comparison of laparoscopic and open partial nephrectomy in clinical T1a renal tumors. J Endourol 2008;22:953-957. 
7. Lifshitz DA, Shikanov SA, Deklaj T, et al. Laparoscopic partial nephrectomy: A single-center evolving experience. Urology 2010;75:282-287.

8. Brandina R, Aron M. Laparoscopic partial nephrectomy: Advances since 2005. Curr Opin Urol 2010;20:111-118.

9. Lane BR, Gill IS. 5-Year outcomes of laparoscopic partial nephrectomy. J Urol 2007;177:70-74.

10. Lane BR, Gill IS. 7-year oncological outcomes after laparoscopic and open partial nephrectomy. J Urol 2010;183: 473-479.

11. Lane BR, Campbell SC, Gill IS. 10-year oncological outcomes after laparoscopic and open partial nephrectomy. J Urol 2013;190:44-49.

12. Lam JS, Shvarts O, Leppert JT, et al. Postoperative surveillance protocol for patients with localized and locally advanced renal cell carcinoma based on a validated prognostic nomogram and risk group stratification system. J Urol 2005; 174:466-472.

13. Levey AS, Bosch JP, Lewis JB, et al. A more accurate method to estimate glomerular filtration rate from serum creatinine: A new prediction equation. Modification of Diet in Renal Disease Study Group. Ann Intern Med 1999;130: 461-470.

14. Matin SF, Gill IS, Worley S, Novick AC. Outcome of laparoscopic radical and open partial nephrectomy for the sporadic $4 \mathrm{~cm}$. or less renal tumor with a normal contralateral kidney. J Urol 2002;168:1356-1360.

15. Richstone L, Montag S, Ost M, et al. Laparoscopic partial nephrectomy for hilar tumors: Evaluation of short-term oncologic outcome. Urology 2008;71:36-40.

16. Mues AC, Okhunov Z, Haramis G, Landman J. Contemporary experience with laparoscopic partial nephrectomy. J Laparoendosc Adv Surg Tech A 2011;21:323-327.

17. Allaf ME, Bhayani SB, Rogers C, et al. Laparoscopic partial nephrectomy: Evaluation of long-term oncological outcome. J Urol 2004;172:871-873.

18. Desai PJ, Andrews PE, Ferrigni RG, Castle EP. Laparoscopic partial nephrectomy at the Mayo Clinic Arizona: Follow-up surveillance of positive margin disease. Urology 2008;71:283-286.

19. Demers EA, Lavelle WF, Cheney R, et al. Charlson versus ASA scores for mortality prediction in patients with vertebral compression fractures. Anesthesiology 2008;109:A976.

20. Mayr R, May M, Martini T, et al. Comorbidity and performance indices as predictors of cancer-independent mortality but not of cancer-specific mortality after radical cystectomy for urothelial carcinoma of the bladder. Eur Urol 2012;62:662-670.

21. Ani I, Finelli A, Alibhai SM, et al. Prevalence and impact on survival of positive surgical margins in partial nephrectomy for renal cell carcinoma: A population-based study. BJU Int 2013;111:E300-E305.

22. Favaretto RL, Sanchez-Salas R, Benoist N, et al. Oncologic outcomes after laparoscopic partial nephrectomy: Mid-term results. J Endourol 2013;27:52-57.

23. Saito $H$, Matsuda $T$, Tanabe $K$, et al. Surgical and oncologic outcomes of laparoscopic partial nephrectomy: A Japanese multi-institutional study of 1375 patients. J Endourol 2012;26:652-659.

24. Jacobs BL, Tan HJ, Montgomery JS, et al. Understanding criteria for the surveillance of patients with a small renal mass. Urology 2012;79:1027-1032.

Address correspondence to: J. Stuart Wolf, Jr., MD, FACS Department of Urology University of Michigan 3875 Taubman Center 1500 East Medical Center Drive Ann Arbor, MI 48109-5330

E-mail: wolfs@umich.edu

$\quad$ Abbreviations Used
ASA $=$ American Society of Anesthesiologists
$\mathrm{CI}=$ confidence interval
$\mathrm{CT}=$ computed tomography
$\mathrm{eGFR}=$ estimated glomerular filtration rate
$\mathrm{HALPN}=$ hand-assisted laparoscopic partial
$\quad$ nephrectomy
$\mathrm{IQR}=$ interquartile range
$\mathrm{MIPN}=$ minimally invasive partial nephrectomy
$\mathrm{MRI}=$ magnetic resonance imaging
$\mathrm{PN}=$ partial nephrectomy
RALPN $=$ robot-assisted laparoscopic partial
nephrectomy
$\mathrm{SD}=$ standard deviation
SLPN $=$ standard laparoscopic partial nephrectomy




\section{This article has been cited by:}

1. De-Hong Cao, Liang-Ren Liu, Yu Fang, Ping Tang, Tao Li, YunJin Bai, Jia Wang, Qiang Wei. 2017. Simple tumor enucleation may not decrease oncologic outcomes for T1 renal cell carcinoma: A systematic review and meta-analysis. Urologic Oncology: Seminars and Original Investigations 35:11, 661.e15-661.e21. [Crossref]

2. Ng Andrew M., Shah Paras H., Kavoussi Louis R.. 2017. Laparoscopic Partial Nephrectomy: A Narrative Review and Comparison with Open and Robotic Partial Nephrectomy. Journal of Endourology 31:10, 976-984. [Abstract] [Full Text HTML] [Full Text PDF] [Full Text PDF with Links]

3. Ho Won Kang, Sung Pil Seo, Won Tae Kim, Yong-June Kim, Seok Joong Yun, Sang-Cheol Lee, Young Deuk Choi, Yun-Sok Ha, Tae-Hwan Kim, Tae Gyun Kwon, Seok-Soo Byun, Seong Uk Jeh, Wun-Jae Kim. 2017. Impact of the ASA Physical Status Score on Adjuvant Chemotherapy Eligibility and Survival of Upper Tract Urothelial Carcinoma Patients: a Multicenter Study. Journal of Korean Medical Science 32:2, 335. [Crossref]

4. Hong-Kai Wang, Xiao-Jian Qin, Chun-Guang Ma, Guo-Hai Shi, Hai-Liang Zhang, Ding-Wei Ye. 2016. Nephrometry scoreguided off-clamp laparoscopic partial nephrectomy: patient selection and short-time functional results. World Journal of Surgical Oncology 14:1. . [Crossref]

5. Jennifer Gordetsky, Michael A. Gorin, Joe Canner, Mark W. Ball, Phillip M. Pierorazio, Mohamad E. Allaf, Jonathan I. Epstein. 2015. Frozen section during partial nephrectomy: does it predict positive margins?. BJU International 116:6, 868-872. [Crossref]

6. Weibin Hou, Zhigang Ji. 2015. Achieving zero ischemia in minimally invasive partial nephrectomy surgery. International Journal of Surgery 18, 48-54. [Crossref] 